Revue d'histoire de l'Amérique française

REVUE D.HISTOIRE DE L'AMÉRIQUE FRANÇAISE

\title{
Le Séminaire de Québec dans le plan de Monseigneur de Laval
}

\section{Lucien Campeau}

Volume 17, numéro 3, décembre 1963

URI : https://id.erudit.org/iderudit/302286ar

DOI : https://doi.org/10.7202/302286ar

Aller au sommaire du numéro

Éditeur(s)

Institut d'histoire de l'Amérique française

ISSN

0035-2357 (imprimé)

1492-1383 (numérique)

Découvrir la revue

Citer cet article

Campeau, L. (1963). Le Séminaire de Québec dans le plan de Monseigneur de Laval. Revue d'histoire de l'Amérique française, 17(3), 315-324.

https://doi.org/10.7202/302286ar d'utilisation que vous pouvez consulter en ligne.

https://apropos.erudit.org/fr/usagers/politique-dutilisation/ 


\section{LE SÉMINAIRE DE QUÉBEC DANS LE PLAN DE MONSEIGNEUR DE LAVAL *}

Ma tâche est de mettre sous vos yeux le rôle que Mgr de Laval a assigné à son séminaire. C'est un devoir écrasant, je ne vous le cache pas. Ce dessein du premier évêque de Québec, à mon sens, est l'un des plus grands qui aient été conçus dans l'Église. Mais de devoir vous l'exposer en quelques minutes, voilà qui double encore la difficulté. Aussi, sans préambule, j'irai à l'essentiel. Je devrai vous faire grâce d'un tableau de cette église naissante, qui serait pourtant utile pour situer le plan du séminaire. Disons seulement que la petite chrétienté française, en 1659, se partage entre trois postes, Québec, TroisRivières et Montréal, et compte environ 2,500 âmes. En 1684, année de la démission de Mgr de Laval, la population aura plus que quadruplé; elle se sera aussi dispersée, s'étendant depuis Rivière-du-Loup jusqu'au-delà de Montréal. Les Jésuites s'occuperont des missions indiennes; les Sulpiciens seront seuls à prendre soin de l'île de Montréal. Le reste des fidèles sera confié à la sollicitude du séminaire de Québec.

C'était, en vérité, la tâche de fonder une église qui s'imposait au prélat. Or l'acte essentiel de cette fondation, ce fut la création du séminaire de Québec. Car pour le premier évêque de Québec, le séminaire, ce sera un clergé, le clergé diocésain de la NouvelleFrance: “... nous avons érigé et érigeons, dès à présent et à perpétuité, un séminaire pour servir de clergé à cette nouvelle église". A ce séminaire-clergé Mgr de Laval confia l'administra-

* Causerie prononcée à Québec, le 17 juin 1963, à l'occasion de la séance inaugurale des fêtes du troisième centenaire du Séminaire de Québec, en présence de l'archevêque de Québec (Son Excellence Mgr Maurice Roy), de nombreux archevêques et évêques et d'un large auditoire composé de religieux, de prêtres et de laïcs. 
tion de tous les biens et revenus qui, en règle normale, assurent la subsistance et l'entretien du clergé dans les autres diocèses. Voilà qui est nouveau. Bien d'autres avant lui, avaient tenté et réussi, dans des instituts particuliers, un retour à l'esprit apostolique de pauvreté. Mais ici, il s'agit d'une refonte de l'organisation ecclésiastique principale du diocèse. Et c'est ce qui fait l'originalité du fondateur. Du même coup, Mgr de Laval apparaît comme un véritable et très grand réformateur de la discipline ecclésiastique. Aussi devrai-je dire un mot de l'histoire de cette discipline, si l'on veut saisir et s'expliquer l'intuition profonde que son œuvre manifeste.

C'est un axiome inviolable, au cours des premiers siècles, que tous les biens d'une église forment une masse indivisible, dont l'administration demeure entre les mains du clergé, du presbyterium indivisible, sous l'unique et personnelle autorité de l'évêque. De même, la distribution des revenus, qui ne peuvent avoir d'autre affectation que le bien de la communauté, est aussi tout entière au pouvoir de la même administration du clergé uni à l'évêque. Toute jouissance personnelle et individuelle des biens ecclésiastiques est sévèrement condamnée. L'église qui forme un tout économique est d'ailleurs toujours, en ce temps, une église épiscopale; nous dirions un diocèse. L'unité d'action du clergé avec l'évêque aboutit même parfois à une pleine communauté de vie quotidienne.

Après la chute de l'empire romain en Occident, l'économie générale devient agricole. Conformément à cette évolution, la meilleure partie des biens productifs de l'Église consiste en terres cultivées, au revenu desquelles continuent de s'ajouter les offrandes des fidèles, dont une partie se fixera en dîmes. En même temps, les charges de l'Église augmentent. Les fidèles se multipliant dans les campagnes qui environnent la ville épiscopale, il faut créer des églises succursales, pourvues chacune d'un clergé et de biens fonciers suffisants pour sa subsistance. Mais l'administration de toutes ces propriétés demeure indivisible, au pouvoir du clergé épiscopal, qui pourvoit sur les revenus communs à l'entretien des clergés des succursales. Par exception, vers le sixième siècle, les évêques concédèrent à quelques clergés 
locaux l'administration des biens de leurs églises et l'usage de leurs revenus. Cette jouissance était si dépendante de la volonté de l'évêque qu'elle était appelée précaire, susceptible d'être enlevée ou rendue à volonté. Au neuvième siècle, pourtant, ces concessions étaient devenues la règle générale. Pendant toute cette première époque, le clerc est considéré comme indéfectiblement attaché à l'église pour laquelle il a été ordonné: cette stabilité est la conséquence de la fidélité à la mission qui lui a été conférée par l'imposition des mains.

En même temps que la propriété ecclésiastique tend à se départager, apparaît une notion juridique qui va révolutionner profondément l'économie de l'Église: j'entends la notion de bénéfice. Le beneficium, selon Thomassin, est à l'origine un bien que l'empereur donne gracieusement aux chefs d'armée et autres militaires, à condition que le bénéficiaire défraie, sur ces revenus, les expéditions qu'il est obligé de faire pour le service de son maître. Parmi ces biens donnés en bénéfice, il y eut des biens ecclésiastiques, qui gardèrent ce titre de bénéfices même après avoir été rendus à des titulaires ecclésiastiques. Peu à peu, le nom arriva à désigner tous les biens fonciers accordés en vue de soutenir un office, aussi bien ecclésiastique que civil, et il signifia aussi l'office lui-même. Ainsi, un épiscopat, une cure devinrent un bénéfice. Alors que les bénéfices civils tendirent à devenir héréditaires, les bénéfices ecclésiastiques, à cause de leur nature même, demeurèrent viagers.

Or le bénéfice, c'est, en premier, une faveur, un bienfait (beneficium), accordé à un individu, même si certaines charges y sont attachées. Alors que la fonction ecclésiastique est d'abord un service de l'Église, les revenus ecclésiastiques n'étant qu'un moyen de soutenir ce service, le bénéficiaire, lui, y voit avant tout un titre à jouir pour son propre compte des avantages, les charges étant une simple condition onéreuse. C'est ainsi que la stabilité de l'office ecclésiastique, qui était autrefois l'expression de la fidélité à une tâche et à une mission sacrées, est vue désormais comme la permanence d'un droit acquis par le titulaire. La propriété et les revenus de l'Église, jalousement divisés entre une infinité de fonctions, plus ou moins strictement liées entre 
elles par le droit, deviennent un champ d'exploitation pour les coureurs de situations faciles. Les abus qui s'ensuivirent sont bien connus: négligence des charges et collection avide des revenus, cumuls de fonctions même inconciliables, népotisme, trafic des offices ecclésiastiques. Les plus criants avaient été corrigés au seizième siècle. Mais il en restait encore assez en France au dix-septième. Plusieurs des plus riches évêchés passaient, par exemple, comme un héritage d'oncle à neveu. C'est surtout dans la disposition des bénéfices inférieurs, spécialement des cures, que le désordre était fréquent. Dans un grand nombre de diocèses, les revenus de la plupart des cures étaient concentrés, inféodés disait-on, entre les mains de quelques gros bénéficiaires, évêques, chapitres, abbayes, seigneurs laïques mêmes, qui retiraient les dîmes, mais faisaient faire le ministère par des prêtres auxquels ils donnaient une pension insuffisante, appelée portion congrue. Le Roi fut obligé, à plusieurs reprises, d'intervenir pour la fixer à un taux raisonnable.

La cupidité est de tous les temps et de tous les milieux et elle peut s'accommoder de toutes les situations juridiques. Mais il faut avouer, je pense, que la notion de bénéfice, selon laquelle une portion des biens de l'Église fructifie, non pour la communauté ecclésiastique, mais pour un individu ou pour une collectivité particulière, la favorise singulièrement et que les correctifs qu'on a pu y apporter ont souvent été inefficaces. De cette première source d'abus découle cette autre, beaucoup moins défendable, que le droit aux revenus ecclésiastiques a pu être séparé du ministère sacré pour lequel il était accordé, en sorte qu'un bénéficiaire pouvait encaisser les fruits d'un office dont un prêtre, comme un mercenaire, portait le fardeau.

L'un des traits les plus lumineux de la figure de Mgr de Laval est l'amour qu'il a porté à ses prêtres; et ses prêtres le lui ont rendu en attachement et en vénération. Il n'a pas voulu les soumettre au péril de devenir des mercenaires dans la vigne du Seigneur; plus encore, afin d'exorciser à jamais la tentation pour quiconque de les plier à ce joug, il a voulu établir dans son église un ordre où la notion même de bénéfice ne trouverait pas d'application, ou du moins serait inopérante. Lui-même nous l'a 
révélé: "il y a tout lieu d'appréhender, écrit-il, ques les évêques qui me succéderont ne prennent la meilleure part de ces dixmes pour eux et qu'ils n'en laissent la moindre aux prêtres qui desserviront les paroisses, comme dans la plupart des diocèses de France, où des prêtres n'ont qu'une pension fort modique, ce qui a bien de mauvais effets..." Un tel langage, déjà, s'oppose à une hypothèse émise par l'abbé Auguste Gosselin, son biographe: "Mgr de Laval, écrit ce dernier, était trop sage et trop prévoyant pour croire que son système de cures, établi conformément à l'édit de 1663 , pouvait avoir un caractère permanent." $\mathrm{Au}$ contraire, le premier évêque de Québec entendait bien construire du définitif. On pourrait, à ce propos, relever nombre de ses expressions qui le prouvent. Contentons-nous de celle-ci: "Je trouve les lettres patentes du Roi nécessaires, parce que, bien qu'elles ne le soient pas pour l'établissement des autres séminaires de France, par l'avis des avocats, néanmoins elles doivent beaucoup affermir ce dépouillement que l'évêque fait du droit qu'il a aux dixmes, afin que les évêques qui nous succéderont ne jugent pas que l'évêque seul les ait liés." Une œuvre à laquelle on s'occupe de donner un fondement légal de cette importance ne peut pas être appelée provisoire.

On aura remarqué, sous la plume du prélat, que la cession des dîmes au séminaire est cela même qu'il veut voir garanti par la confirmation royale; et c'est aussi ce qui est le plus fortement souligné dans les lettres patentes. C'est qu'en principe, la dîme est la principale pourvoyeuse de la subsistance du clergé. En fait, elle sera bien loin de suffire, durant tout le régime français, et surtout au temps de Mgr de Laval. Comme le séminaire devait contenir bien d'autres œuvres que les paroisses, l'évêque dut lui assurer encore diverses sources de revenus, sous forme de dotations en biens fonciers ou en rentes, de subventions royales et d'autres aumônes. L'important est de savoir que la totalité des moyens de subsistance du clergé diocésain en Nouvelle-France était au pouvoir du séminaire. Il n'y eut que le bénéfice épiscopal qui demeura distinct, parce qu'il n'était pas au pouvoir de l'évêque de le réformer. Et même, après avoir entièrement renoncé aux dîmes et cédé en bonne partie au séminaire, sous 
forme de dotation du chapitre, les revenus épiscopaux, Mgr de Laval lui abandonna encore ses biens personnels. En fait, vivant dans la communauté du séminaire, il ne garda à peu près rien de ses revenus propres durant son épiscopat. En sorte qu'en pratique le bénéfice épiscopal lui-même fut réuni à l'œuvre du clergé diocésain. Le désintéressement fut poussé au point que le prélat s'interdit à lui-même et à ses successeurs, non seulement tout usage particulier des revenus de son église, mais encore la faculté d'en aliéner les fonds, même en cas de nécessité, sans le consentement des quatre principaux officiers du séminaire.

Qu'est-ce, maintenant, que ce séminaire, pour lequel le prélat a eu tant d'attentions ? C'est, dans sa pensée, une école de formation des jeunes clercs, conforme aux décrets du concile de Trente; mais il contiendra aussi un chapitre, formé de prêtres de la même institution; et il sera en outre "une continuelle école de vertu et un lieu de réserve" d'où l'évêque tirera des prêtres pour remplir tous les offices du diocèse. En un mot, le séminaire de Québec, c'est un clergé diocésain complet, avec tous ses degrés, ses institutions et ses fonctions ordinaires, aussi complet que celui des églises épiscopales de l'antiquité. Ce clergé se trouve encore érigé en un véritable institut de vie commune, en une authentique communauté ecclésiastique. Malgré tout, ce n'est ni un ordre, ni une congrégation; ce n'est pas non plus une société de prêtres séculiers réunis en vue d'une mission particulière; c'est et cela demeure le clergé diocésain, appliqué par l'ordination ou l'incardination à tous les besoins du diocèse. Il n'est fait aux prêtres aucune obligation d'y entrer; il n'est demandé à aucun d'abdiquer ses possessions personnelles, bien que plusieurs de ses membres, et l'évêque le premier, l'aient fait spontanément. Cette libéralité est comme une extention de la désappropriation, du dépouillement de l'esprit de propriété, qui est exigée comme une attitude fondamentale à l'égard de tous les revenus du diocèse. Cette communauté met à la disposition de ses membres des moyens de sanctification très efficaces; elle est "une continuelle école de vertu". Mais elle se charge aussi de tous les besoins temporels de tous les associés jusqu'à leur retour en France ou jusqu'à leur mort, selon le cas. 
Aussi, dans ce séminaire, n'y a-t-il plus de bénéfice individuel. C'est pourquoi Mgr de Laval ne veut pas entendre parler de curé inamovible. Le curé inamovible, par nature, c'est le curé bénéficiaire, auquel le droit aux revenus de la paroisse ne peut être enlevé que par la mort ou par un procès canonique. Ses prêtres à lui seront donc amovibles. Ce n'était pas caprice d'un esprit autoritaire qui entendait jouer avec ses prêtres comme avec les pièces d'un échiquier; mais la notion même de bénéfice s'opposait à l'idée que le fondateur se faisait primitivement de son séminaire. Il est important de noter ici que Mgr de Saint-Vallier n'entendra pas l'inamovibilité au même sens que son prédécesseur. Est-ce à dire qu'évitant l'écueil des bénéfices individuels, le fondateur créait quand même un gros bénéficiaire collectif dans le séminaire ? Quand cela serait, ce n'eût été qu'un moindre mal; car le séminaire était le clergé même qui remplissait tous les ministères du diocèse. Mais $\mathrm{Mgr}$ de Laval évitait encore cet inconvénient en disposant à l'avance des surplus de l'institution, qu'il affecta à la construction des églises fardeau normal des fabriques, non des curés - et aux diverses œuvres qu'il faut développer dans un diocèse. Ainsi était-il assuré que tous les biens et tous les revenus du clergé seraient intégralement employés à l'avantage commun du diocèse, sans procurer l'enrichissement ni d'un individu, ni d'une collectivité.

La première pensée de Mgr de Laval semble avoir été de rattacher au séminaire toutes les cures qu'il n'avait pas laissées aux Jésuites et aux Sulpiciens. Le séminaire aurait été ainsi le curé de toutes et les aurait desservies par ses membres. Les premières paroisses furent érigées de la sorte. Mais l'état du pays empêchait encore l'établissement de curés résidents. L'évêque savait mieux que le gouverneur et l'intendant qu'un curé doit résider parmi ses ouailles et il le souhaitait bien plus sincèrement qu'eux. De tous les lieux qui dépendaient du séminaire, la paroisse de Québec exceptée, celui qui comptait le plus fort groupe de chrétiens, en 1683, était Charlesbourg, avec 397 âmes. On comptait en tout 69 localités habitées, de Rivière-du-Loup jusqu'à l'île Jésus, et leur population moyenne était de 95 âmes. 
Certaines n'avaient que quatre et même qu'un seul habitant. Comme le séminaire ne disposait alors que de quinze prêtres pour les desservir, il est bien évident qu'il ne pouvait y avoir, à la fin de l'épiscopat de Mgr de Laval, de mauvaise volonté chez l'évêque à propos des cures fixes. Ce terme de cures fixes, d'ailleurs, est d'une polyvalence telle qu'il peut couvrir toutes les confusions et toutes les intrigues. Quand les habitants réclament un curé fixe, ils ne veulent qu'un prêtre résident; le malheur est qu'ils refusent de le faire vivre. Quand l'intendant parle de cure fixe, il désire appliquer les édits royaux. Le Roi, en effet, dans l'intention de procurer la sécurité aux prêtres des paroisses, ordonnait de n'y établir que des curés bénéficiaires ou des vicaires auxquels on garantissait la perpétuité de leur vicariat. Les édits ne s'appliquaient pas en Nouvelle-France, puisque tous les prêtres y avaient leur subsistance assurée jusqu'à la mort. Et si les légistes, férus de la lettre de la loi, voulaient insister, Mgr de Laval pouvait le plus facilement du monde considérer ses curés comme des vicaires perpétuels. La difficulté n'était pas là; elle était de trouver des prêtres et des cures rentables. Mais Frontenac, qui s'était juré de détruire l'œuvre de Mgr de Laval, entendait par curé fixe un prêtre qui aurait rompu tous les liens avec le séminaire. Ce gouverneur autocrate et passionné, qui s'est acquis un grand nom sans avoir jamais eu une seule grande pensée, n'avait évidemment aucune aptitude pour comprendre le dessein du prélat et il ne cessait d'envoyer au ministre et au Roi des rapports incomplets, tendancieux ou simplement faux.

Plût à Dieu que Frontenac ait obéi aux ordres du Roi comme Mgr de Laval le faisait de son côté. Devant l'insistance royale sur l'établissement des cures fixes, devant la pauvreté et la mauvaise volonté des habitants qui demandaient des curés résidents et faisaient difficulté pour les entretenir, devant la malveillance incurable et obtuse du gouverneur, le prélat se résolut à renoncer en fait aux avantages des lettres patentes de 1663 et à établir les cures sur le même pied qu'en France. Il remit en entier la dîme et les autres offrandes aux curés. C'était créer des curés bénéficiaires, donc des curés fixes au sens le plus parfait du mot. Et voilà des curés inamovibles, créés par Mgr 
de Laval lui-même; il y en eut neuf dans ce cas. La mesure, toutefois, ne pouvait guérir le mal, dont la source se trouvait dans l'état économique du pays et dans le manque de prêtres. L'évêque, malgré cette importante concession, avait trouvé le moyen de sauver son plan de réforme. Au lieu d'être obligatoire, comme auparavant, l'union des cures au séminaire fut libre. Les curés eux-mêmes, qui sortaient du séminaire, se chargèrent de la préserver. Ils rendirent compte, selon le règlement du séminaire, de toutes les recettes de leurs bénéfices et le séminaire continua de garantir leur complète subsistance. Ils y trouvaient d'ailleurs leur avantage temporel, parce que la plupart des cures ne pouvaient les faire vivre; mais les intérêts spirituels qu'ils retiraient du séminaire étaient un motif encore plus puissant à leur esprit. Aucun prêtre ne fit défection à Mgr de Laval. Et cette forme d'union libre des cures, inaugurée en 1678, subsista jusqu'à 1692, année où Mgr de Saint-Vallier, s'alliant avec Frontenac, réussit à disloquer l'édifice, à la grande douleur du clergé canadien.

En terminant, on peut se demander si l'œuvre du séminaire, telle que Mgr de Laval l'a conçue, était durable. Même les historiens favorables ne le croient pas aujourd'hui. Pourtant, le fondateur l'avait voulue permanente et je pense, pour ma part, qu'elle pouvait durer. Autant que je comprenne son intention, Mgr de Laval entendait restaurer le primat de la charité, plutôt que celui de la loi, dans l'organisation ecclésiastique. S'il faut admettre qu'un tel effort est condamné à l'échec dès le principe, que faudra-t-il dire de cette gageure, bien plus vaste encore, qu'est le christianisme lui-même? Il n'y avait pas d'obstacle insurmontable à la survie de l'œuvre du séminaire. Elle n'était pas en désaccord avec les lois du royaume, grâce à la sagesse et à l'esprit de conciliation de son fondateur. Le clergé lui-même y était entièrement gagné, tellement Mgr de Laval avait su lui communiquer son propre esprit. Il n'a manqué qu'un successeur animé des mêmes sentiments, pour continuer la tâche. Si le grand évêque avait pu, durant les vingt-quatre années qui lui restaient à vivre, affermir l'institution et la conduire à maturité, ou bien s'il avait pu la confier à l'un de ces hommes remarquables qu'il avait formés et qui l'ont si bien 
secondé, le séminaire, en plus de l'œuvre glorieuse qu'il a accomplie dans le champ de la formation ecclésiastique et de l'éducation, ne pourrait-il pas encore présenter à l'ensemble de l'Église un type d'organisation diocésaine qui serait à la fois un exemple et un sujet d'admiration pour le reste de la chrétienté ?

LUCIEN CAMPEAU, s.j. 